The Bulletin of Symbolic Logid

Volume 18, Number 3, Sept. 2012

\title{
IN MEMORIAM: ERNST SPECKER
}

1920-2011

Born in Zurich, Switzerland, February 11, 1920, Ernst Paul Specker spent most of his life in that city, contributing decisively to the continuing international renown of the ETH in mathematics and logic. He died December 10, 2011. Set back by an early illness, he entered the ETH late but then, as a brilliant student, got his degree with the famous topologist and geometer Heinz Hopf with a thesis on cohomology and covering complexes in three dimensions, corresponding, so Specker, to the Zeitgeist. Between this publication in 1949 and his last one in 2011 on a generalized chess problem, the scope of Specker's work is impressively diverse in subject matter. But there is a coherence that stems from a characteristic taste in the selection of topics and problems, and in the ingenuity of invention and construction: Specker was attracted by outstanding mathematical challenges; as a young scientist by deep questions in the foundations of mathematics and physics; by combinatory set theory, and by interchange and collaboration originating mostly from his own work.

Recursiveness, by the 1940's, had established itself as the preferred mathematical candidate for the notion of constructivity. The foundational challenge was to determine differences between classical mathematics, in particular analysis, and 'constructive' analogues. The existence of such differences had been held at the time, on completely different grounds, by the intuitionists. Specker [3]: There are bounded monotone recursive sequences of rational numbers (now called Specker sequences), which do not converge to a recursive real number. The proof illustrates the fact that there are theorems of classical analysis that are not constructively provable. Again Specker [4]: There are continuous recursive real functions whose maximum on the closed unit interval is not itself recursive. These are seminal papers of a now well-developed field.

Model Theory in the early 1950's was, especially in the view of Tarski and others, the logically adequate form of the axiomatic standpoint emerging in the first half of that century. The challenge is to find general phenomena in the relation between formal axiomatic theories and their models. Specker [5]: Formal axioms may admit groups of transformations; do corresponding models admit corresponding (auto)morphisms? This connection is far from trivial even for groups of order 2: duality in projective geometry (interchanging 'point' and 'line' in the axioms, versus polarity in projective 
planes.) Infinite transformation groups on formal axioms arise in 'ambiguous type theory', a formal version of simple type theory (which considers a sequence of types of sets where the next type includes all subsets of sets of the previous type). Ambiguous type theory formally admits types of all integer orders (including negative types) by introducing corresponding variables $x^{t}, y^{t}, t \in \mathbb{Z}$ and where an (sub-)expression $x^{s} \in y^{t}$ is only legal if $s=t-1$. The comprehension axiom introduces sets of the next type as expansion of legally formulated properties of sets of a given type. This theory is related to Quine's New Foundations NF. Specker [6]: NF is consistent iff there is a model of ambiguous type theory which admits automorphisms moving types by one level.

Foundations of Set Theory, in the time between Gödel and Cohen, had many fundamental loose ends: the sustained attempt by Rosser and others to use NF as a basis of a 'logic for mathematicians' put a premium on relating this rather formally motivated theory with our understanding of naïve set theory on which contemporary mathematics relies. Specker [7]: The axiom of infinity is provable in NF but the axiom of choice and the continuum hypothesis are false. The proof (three pages, communicated to the Proceedings of the National Academy of Sciences by Hermann Weyl, with Specker at The Institute of Advanced Studies at the time), is a beautiful example of its authors exceptional formal ingenuity. Specker remained ambivalent about the consistency of NF, but never completely lost interest in the challenge of NF; he subsequently focused on consistency problems in the context of the more plausible von Neumann-Bernays set theory Specker [8], [9]: The independence of the axiom of foundations (the stumbling block in pre-Cohen proofs of the independence of the axiom of choice for full Zermelo-Fraenkel set theory). Development of some alternatives to this axiom and its relations to the continuum hypothesis.

Combinatorial Problems are an inexhaustible source of mathematical challenges and striking phenomena, especially in combinatory set theory. Specker [10]: Let $R$ be a binary relation on a countably infinite set $S$, itself consisting of a countably infinite number of disjoint infinite subsets $S_{i}$. Then for each finite number $n$ there exists either a subset $S^{\prime}$ of size $n$ of $S$ all of whose elements are in the relation $R$, or there exists a subset $S^{\prime \prime}$, none of whose elements are in the relation $R$, and which intersects each $S_{i}$ infinitely often. This generalization of Ramsey's theorem, now usually denoted by $\omega^{2} \rightarrow\left(\omega^{2}, n\right)^{2}, 2<n<\omega$, was posed as a problem by Erdös, who calls Specker's proof an ingenious argument. However $\omega^{2} \rightarrow\left(\omega^{2}, 4\right)^{3}$ does not hold; one of many more results on partition relations in this seminal paper.-Collaboration with Erdös [11] led to another striking result, one of whose consequences is the following: Assuming the continuum hypothesis, given a well-ordering of the reals and a subset $A$ of the (real) plane such that every horizontal line intersects it on a set of order type $\beta<\omega_{1}$ then the complement of $A$ contains a square of the size of the continuum. 
Nobody really understand quantum theory, as some of my friends in theoretical physics maintain, tongue only partly in cheek. I understand this to mean that while the logic of combining observations in classical physics is the usual classical one, this is not so in the world of quanta. Quantum Logic, initiated in the 1930's by von Neumann, relates subspaces of the (unitary) Hilbert spaces of quantum mechanics, understood as 'propositional observables', to elements of a logical system. This led to some controversies. Specker [12] refined the approach by including the important notion of commensurability (reflecting the fact that not all observations are commeasurable), with the result that the operations that give rise to quantum logic are no longer total, and therefore lead to partial Boolean lattices. In collaboration with Kochen [13], [14] the challenge of hidden variables was taken up again and solved in this framework. A concrete physical experiment is proposed for testing the model.-The way in which Specker internalized the problematics of quantum theory is beautifully illustrated in his first paper by a charming tale of a princess in a far country.

Complexity Theory stands out among the many challenges that computer science brought to mathematics. The focus changed from computability and decidability 'in principle' (by recursive functions) to feasibility (by polynomially computable functions), a concept which turns out to be just as stable epistemologically as the first one. The computational problem closest to logic concerns propositional formulas. Specker, in collaboration with Hodes [15] determines lower bounds on the growth of formulas when (propositional) quantifiers are eliminated. Together with Lieberherr [16], he investigates relations between the structure of such formulas and their computational complexity. But Specker's main collaboration was with his students such as Martin Fürer and particularly with his friend Volker Strassen with whom he conducted an influential seminar [17] on a broad selection of topics in complexity.

Inevitably, people were attracted to Specker and loved to work and relax with him. In both contexts he provided an atmosphere of broad mathematical and general culture and a charming way of dealing with questions, mathematical, personal and ethical. These friendships started early in the Bernays Seminar with visitors from abroad: Hao Wang, William Craig, Roman Sikorski, Robert Vaught, and others. Most interactions resulted in joint papers, dealing with a variety of topics, too numerous to mention. But in each of them we discern unguem leonis, for example in the influential paper with MacDowell [18]: Group theoretic, measure theoretic and combinatorial notions and results are employed to investigate the structure of nonstandard models of arithmetic.- Other collaborators are referred to above, but the picture would be sorely incomplete without mentioning Gaifmann, Coray, Makowsky, Blatter, Scarpellini, Hungerbuehler, Zachos, and most recently Schlude 2011. 
Specker's scientific work lives on not only in his publications, in their many and important sequels, but most vividly in our memories.-There were four international conferences dedicated to his work, on the occasion of his last round birthdays. The proceedings [1] for the 60th is introduced by a charming biography written by Hao Wang. A collection [2] of Specker's papers to 1990, the 'Selecta', contains a biographical contribution by Jonas Meon; the style reveals this to be a pseudonym. All of the papers cited above are reprinted in [2].

Erwin Engeler

\section{REFERENCES}

[1] Logic and Algorithmic, An International Symposium held in Honour of Ernst Specker, (E. Engeler, H. Läuchli and V. Strassen, editors), L'Enseignement Mathématique, Geneva, 1982. 392 pp.

[2] Ernst SPecker, Selecta, with a preface by Gerhard Jäger, Hans Läuchli, Bruno Scarpellini and Volker Strassen, editors; with a biography of Ernst Specker by Jonas Meon, Birkhäuser Verlag, Basel, 1990.

[3] Nicht konstruktiv beweisbare Sätze der Analysis, The Journal of Symbolic Logic, vol. 14 (1949), pp. 145-158.

[4] Der Satz vom Maximum in der rekursiven Analysis, Constructivity in Mathematics, Proceedings of the colloquium held at Amsterdam, 1957, (A. Heyting, editor), pp. 254-265, Studies in Logic and the Foundations of Mathematics, North Holland Publishing Company, Amsterdam 1959.

[5] Dualität, Dialectica, vol. 12, pp. 451-465.

[6] Typical ambiguity, Logic, Methodology and Philosophy of Science, Proceedings of the 1963 International Congress, (Y. Bar-Hillel, editor), pp. 45-57, Studies in Logic and the Foundations of Mathematics, North Holland Publishing Company, Amsterdam 1965.

[7] The Axiom of choice in Quine's New Foundations for Mathematical Logic, Proceedings of the National Academy of Sciences of the United States of America, vol. 39 (1953), pp. 972975.

[8] Verallgemeinerte Kontinuumshypothese und Auswahlaxiom, Archiv der Mathematik, vol. 5 (1954), pp. 332-337.

[9] Zur Axiomatik der Mengenlehre (Fundierungs- und Auswahlaxiom), Zeitschrift für mathematische Logik und Grundlagen der Mathematik, vol. 3 (1957), pp. 173-210.

[10] Teilmengen von Mengen mit Relationen, Commentarii Mathematici Helvetici, vol. 31 (1957), pp. 302-314.

[11] (with P. Erdös), On a theorem in the theory of relations and a solution to a problem of Knaster, Colloquium Mathematicum, vol. 8 (1961), pp. 19-21.

[12] Die Logik nicht gleichzeitig entscheidbarer Aussagen, Dialectica, vol. 14 (1960), pp. 239-246.

[13] (with Simon Kochen), Logical structures arising in quantum theory, The Theory of Models, Proceedings of the 1963 International Symposium at Berkeley, (J. W. Addison, L. Henkin, and A. Tarski, editors), pp. 177-189, Studies in Logic and the Foundations of Mathematics, North Holland Publishing Company, Amsterdam 1965.

[14] (with Simon Kochen), The problem of hidden variables in quantum mechanics, Journal of Mathematics and Mechanics, vol. 17 (1967), pp. 59-88. 
[15] (with L. Hodes), Length of formulas and elimination of quantifiers, Contributions to Mathematical Logic; Proceedings of the Logic Colloquium, Hannover 1967, (H. A. Schmidt, K. Schütte, and H.-J. Thiele, editors), pp. 175-188, Studies in Logic and the Foundations of Mathematics, North Holland Publishing Company, Amsterdam 1968.

[16] (with K. Lieberherr), Complexity of partial satisfaction, Journal of the Association of Computing Machinery, vol. 28 (1979), pp. 411-421.

[17] (with V. Strassen), Einleitung, Komplexität von Entscheidungsproblemen, (E. Specker und V. Strassen, editors), pp. 1-10, Lecture Notes in Computer Science, Springer Verlag, Heidelberg 1976.

[18] (with R. MacDowell), Modelle der Arithmetik, Infinitistic Methods, Proceedings of the Symposium on Foundations of Mathematics, Pergamon Press, London, 1961, pp. 257-263. 